\title{
Ultraviolet extinction toward a quiescent molecular cloud in the Small Magellanic Cloud
}

\author{
J. Maíz Apellániz ${ }^{1}$ and M. Rubio² \\ 1 Instituto de Astrofísica de Andalucía-CSIC, Glorieta de la Astronomía s/n, 18008 Granada, Spain \\ e-mail: jmaiz@iaa.es \\ 2 Departamento de Astronomía, Universidad de Chile, Casilla 36-D, Santiago, Chile \\ e-mail: monica@das.uchile.cl
}

Received 22 December 2011 / Accepted 27 January 2012

\begin{abstract}
Context. The mean UV extinction law for the Small Magellanic Cloud (SMC) is usually taken as a template for low-metallicity galaxies. However, its current derivation is based on only five stars, which renders its universality questionable. More targets with measured extinction laws in the SMC are necessary to determine its possible dependence on parameters such as metallicity and starforming activity.

Aims. We aim to measure the UV extinction law for several stars in the quiescent molecular cloud SMC B1-1.

Methods. We obtained HST/STIS slitless UV spectroscopy of a $25^{\prime \prime} \times 25^{\prime \prime}$ field of view and combined it with ground-based NIR and visible photometry of the stars in the field. The results were processed using the Bayesian photometric package CHORIZOS to derive the visible-NIR extinction values for each star. The unextinguished spectral energy distributions (SEDs) obtained in this way were then used to derive the UV extinction law for the four most extinguished stars. We also recalculated the visible-NIR extinction for the five SMC stars with preexisting UV extinction laws.

Results. The UV extinction law for four SMC B1-1 stars that are situated within several pc of each other differs significantly from star to star. The $2175 \AA$ fump is moderately strong in one, weak in two, and absent in the fourth.
\end{abstract}

Key words. dust, extinction - Magellanic Clouds - ultraviolet: ISM - stars: early-type

\section{Introduction}

In the 1980s the International Ultraviolet Explorer (IUE) was extensively used to derive the UV extinction law for a large number of stars in the Milky Way and the Magellanic Clouds. It became clear then that there were considerable variations among sightlines in the three galaxies. Cardelli et al. (1989) used several tens of stars to derive a one-parameter family of extinction laws for the Galaxy. There were even fewer useful targets for the Large Magellanic Cloud (LMC) and still less for the Small Magellanic Cloud (SMC). The often quoted SMC extinction law of Prévot et al. (1984) was based in only three stars (AzV 398, $\mathrm{AzV}$ 18/Sk 13, and Sk 191). Sk 191 turned out to be a poor choice since it is actually an unreddened object, which left only two useful objects. The re-analysis of Gordon \& Clayton (1998) added two more targets, AzV 214 and AzV 456/Sk 143, and Gordon et al. (2003) completed the current list of SMC stars with a derived UV extinction law with a fifth target, AzV 23/Sk 17.

The characteristic that is most often quoted about the SMC extinction laws is the absence of the $2175 \AA$ wide absorption bump, which in the Fitzpatrick \& Massa (1990) parameterization translates into a zero or very small $c_{3}$ term. However, this is not true of all SMC extinction laws since AzV 456/Sk 143 clearly shows the absorption feature. The different location of $\mathrm{AzV}$ 456/Sk 143 in the SMC "wing" compared with that of the other stars in the SMC bar prompted Gordon \& Clayton (1998) to compute two different SMC extinction laws, one for the wing and one for the bar, based on one and three ${ }^{1}$ stars, respectively. The results of Gordon et al. (2003) also suggest another possible difference between the two regions: their measured $R_{V}$ values for the four bar stars are in the typical range of 2.40-3.30 while that of the wing star is, $2.05 \pm 0.17$. The existence of these detected variations in a small sample is an obvious sign for the need for a better sample to quantify the prevalence of the different extinction laws and to understand the underlying reason for these variations. Another reason why a better sample is needed are the relatively low values of $E(B-V)$ for the five stars, 0-150.26 (Gordon et al. 2003), which cause large uncertainties in the measured $R_{V}$ values.

Why should we care about the SMC extinction law? In addition to the importance that it has by itself, because the SMC is our best template for low-metallicity galaxies. Indeed, it was assumed for some time that the absence of the $2175 \AA$ bump in the SMC extinction law was a metallicity effect but the detection of such a feature in Azv 456/Sk 143 seems to invalidate that hypothesis. Several explanations are possible, such as the existence of metallicity gradients in the ISM or the effect of "starformation activity", a term that refers to the possible destruction of dust grains by UV radiation or shocks from massive stars. Until we have a better sample, we will likely not be able to decide which explanation is the most plausible one.

\footnotetext{
1 As we have already mentioned, Gordon et al. (2003) added another star, AzV 23/Sk 17, also located in the bar.
} 
We selected as our target SMC B1-1, a cold molecular cloud without signs of star formation that is located at the southern end of the SMC bar. It has a molecular mass of $2.4 \times 10^{4} M_{\odot}$ (Rubio et al. 2004) as derived from the $\mathrm{CO}(1-0)$ emission line and its virial mass was determined using observations made with the Swedish ESO Submillimeter Telescope (SEST) at La Silla Observatory. Of the stars with existing UV extinction laws, the closest one to SMC B1-1 is AzV 18/Sk 13, located $\sim 14^{\prime}$ away.

\section{Observations and data description}

\subsection{STIS data}

SMC B1-1 was observed with the Space Telescope Imaging Spectrograph (STIS) onboard the Hubble Space Telescope (HST) on 27 May 2004. We obtained four imaging exposures with STIS/NUV-MAMA, two imaging exposures with STIS/FUV-MAMA, and one slitless spectral exposure with STIS/NUV-MAMA. Both MAMA detectors have no read noise and, in imaging mode, cover a field of $25^{\prime \prime} \times 25^{\prime \prime}$ with $1024 \times 1024$ pixels. The NUV imaging exposures used two different filters, F25CN270 (centered at $2702 \AA$, see Fig. 1) and F25CN182 (centered at $1895 \AA$ ) with total exposures times of $100 \mathrm{~s}$ for each filter. The FUV imaging exposures used a single filter, F25QTZ (centered at $1559 \AA$ ) with a total exposure time of $200 \mathrm{~s}$. The slitless spectral exposures, which cover an area slightly bigger than that of the images, were obtained with the NUV objective prism (Fig. 2) with a total exposure time of $916 \mathrm{~s}$. The NUV objective prism has a variable dispersion that goes from $\approx 5 \AA /$ pixel at $1750 \AA$ to $\approx 45 \AA /$ pixel at $3000 \AA$ (STScI 2007). Our spectral exposures were obtained in the shadowed part of the orbit and with the F25SRF2 filter to minimize the effect of the geocoronal background.

There are 15 stars that are easily detected in all three UV imaging filters and in the spectral exposures. They have been labeled in Figs. 1 and 2 in order of ascending $y$ coordinate. Note that the stars were not placed on an absolute reference frame, so it is possible that the RA+Dec grid is offset by $\sim 1^{\prime \prime}$, as is typical with coordinates derived from the header information in HST images.

\subsection{NIR imaging}

Deep NIR $J H K_{\mathrm{s}}$ imaging was obtained with ISAAC at the $8.2 \mathrm{~m}$ VLT telescope at Paranal Observatory on 24-25 September 1999 (63.C-0329(A)). We used the short wavelength arm equipped with a $1024 \times 10241024$ pixels Hawaii Rockwell array, with a spatial resolution of $0 .{ }^{\prime} 148^{\prime \prime} /$ pix and a total field of view of $2.5 \times 2.5$.

The observations were made in a series of six frames (NDIT), each individual frame with a $10 \mathrm{~s}$ integration time (DIT) in each filter. The individual frames were coadded and a $60 \mathrm{~s}$ image was stored. A ten position dither mosaic was made with a separation of $15^{\prime \prime}$ using the same observing strategy to cover the SMC B1-1 region. In each filter, we interleaved sky frame observations for every $10 \mathrm{~min}$ of on-source imaging. These sky frames were chosen in a field with faint stars and no extended emission located $300^{\prime \prime}$ south of the position of SMC B1-1. The sky field was observed in a similar way as the source frames. The procedure was repeated until we achieved a final integration time of $3600 \mathrm{~s}$ in $J, H$, and $K_{\mathrm{s}}$.

To produce the final images, each image was dark-corrected, flat-fielded, and sky-subtracted, and then median-averaged and
Table 1. ISAAC/VLT NIR photometry.

\begin{tabular}{lccc}
\hline \hline Star & $J$ & $H$ & $K_{\mathrm{s}}$ \\
\hline 01 & $19.414 \pm 0.069$ & $19.402 \pm 0.072$ & $19.525 \pm 0.094$ \\
02 & $20.002 \pm 0.072$ & $20.090 \pm 0.074$ & $19.968 \pm 0.104$ \\
03 & $20.086 \pm 0.073$ & $20.006 \pm 0.076$ & $19.963 \pm 0.100$ \\
04 & $19.864 \pm 0.038$ & $19.797 \pm 0.045$ & $19.647 \pm 0.041$ \\
05 & $18.836 \pm 0.066$ & $18.740 \pm 0.068$ & $18.718 \pm 0.082$ \\
$06+07$ & $17.987 \pm 0.065$ & $17.994 \pm 0.068$ & $18.000 \pm 0.079$ \\
08 & $18.436 \pm 0.066$ & $18.317 \pm 0.068$ & $18.235 \pm 0.080$ \\
09 & $18.424 \pm 0.066$ & $18.378 \pm 0.068$ & $18.307 \pm 0.081$ \\
10 & $18.812 \pm 0.066$ & $18.759 \pm 0.068$ & $18.739 \pm 0.082$ \\
11 & $18.464 \pm 0.067$ & $18.400 \pm 0.069$ & $18.426 \pm 0.083$ \\
12 & $20.246 \pm 0.055$ & $19.382 \pm 0.035$ & $18.877 \pm 0.041$ \\
13 & $19.402 \pm 0.024$ & $19.400 \pm 0.034$ & $19.258 \pm 0.045$ \\
14 & $17.658 \pm 0.065$ & $17.165 \pm 0.067$ & $17.066 \pm 0.078$ \\
15 & $17.236 \pm 0.065$ & $16.681 \pm 0.067$ & $16.566 \pm 0.077$ \\
\hline
\end{tabular}

combined using IRAF procedures. The final images were registered with respect to the $J$ image by means of several common stars. The final ISAAC/VLT images cover a $2.5 \times 55^{\prime} 0$ area and have limiting magnitudes of about $J=21, H=21$, and $K_{\mathrm{s}}=22$, respectively.

We identified the 15 stars observed in the HST field in the VLT/NIR images and performed aperture photometry using the IRAF/DAOPHOT package. We used the standard stars observed on both nights to calibrate the photometry in $K_{\mathrm{s}}$ (observed the first night) and in $H$ and $J$ (observed the second night). Additionally, we selected several stars from the 2MASS IR images with good photometry (AAA label) and compared their catalog magnitudes with our $J H K_{\mathrm{s}}$ photometry. The agreement between our photometry and 2MASS is good to $0.01 \mathrm{mag}$. The NIR photometry in given in Table 1. Two of the stars (06 and 07) are too close together in the ground-based data to be resolved, so unresolved magnitudes are provided.

\subsection{Visible photometry}

The Johnson-Cousins $U B V I$ photometry of the sources present in the STIS exposures was obtained from Zaritsky et al. (2002) using the VizieR (Ochsenbein et al. 2000) J/AJ/123/855 catalog. 13 of the 15 stars are resolved in the Zaritsky et al. (2002) data while the pair 06+07 appears as a single unresolved source.

\section{Processing of the SMC B1-1 data}

Our goal in this paper is to obtain the UV extinction law of as many as possible of the stars in the SMC B1-1 region for which we can measure their spectra. The strategy to accomplish that is:

1. measure the effective temperature $\left(T_{\text {eff }}\right)$ and optical/NIR extinction of the 14 sources that are point-like in the groundbased photometry;

2. extract the spectra for the 15 point sources in the NUV slitless exposures;

3. select the sources with the highest optical/NIR extinction, since for those objects the UV extinction should be more easily measurable;

4. calculate the extinction law $k(\lambda)$ for the stars selected in the previous step.

\subsection{Effective temperatures and optical color excesses}

The traditional mechanism to measure UV extinction laws is known as the pair method (Massa et al. 1983) and requires 
J. Maíz Apellániz and M. Rubio: UV extinction toward a quiescent molecular cloud in the SMC

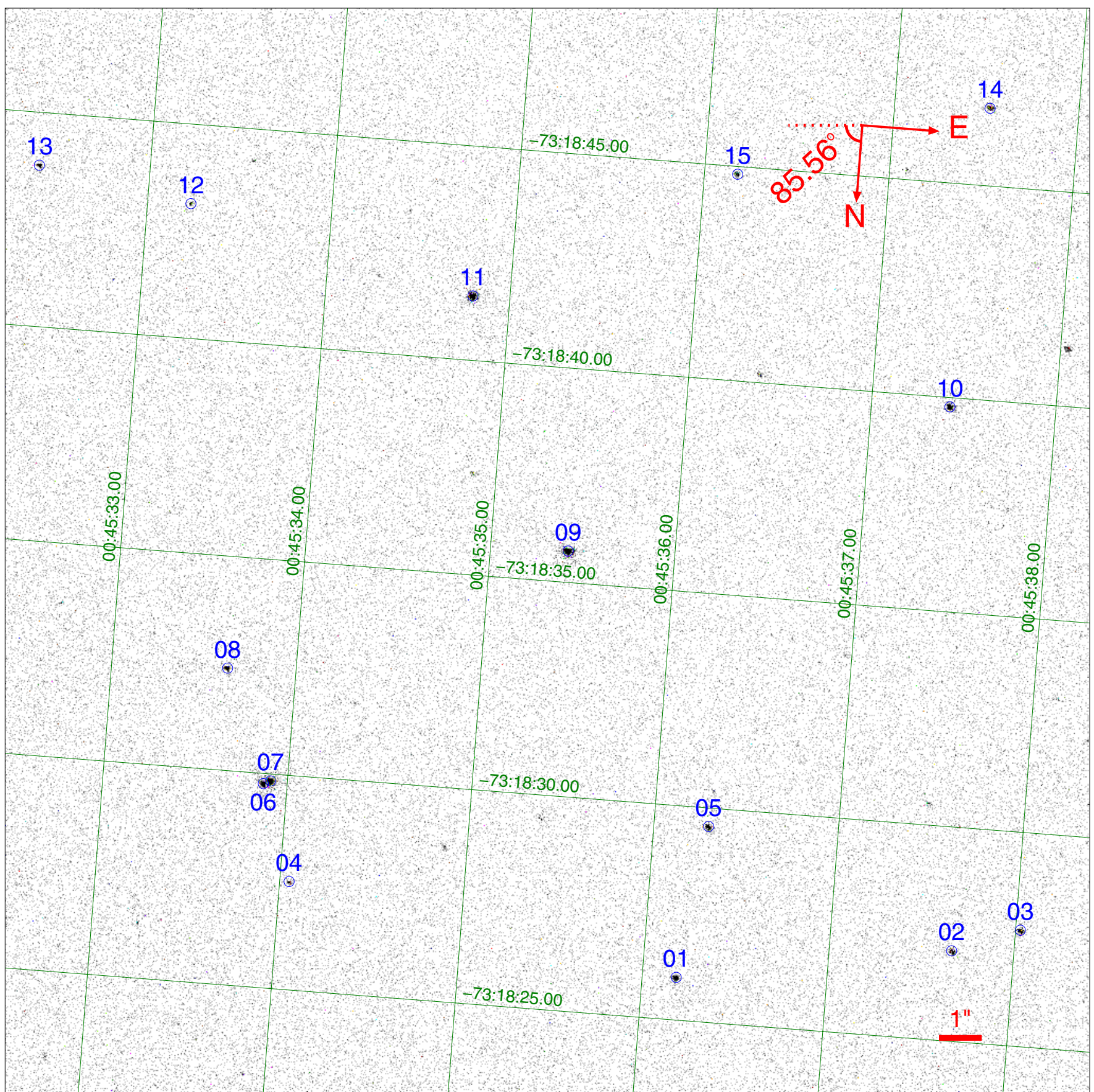

Fig. 1. F25CN270 STIS NUV-MAMA image of the SMC B1-1 region. The stars for which photometry was extracted are labeled.

obtaining spectra of two stars of the same spectral type, one with high extinction and the other one with low extinction. The fluxes of the two stars are corrected for distance effects if necessary, the low-extinction spectral energy distribution is divided by the high-extinction one, and the result is normalized by the extinction difference between the two. The pair method requires the accurate measurement of the spectral type of the extinguished star, which may not be straightforward for dim objects, and the availability of data for a star with the same spectral type, which can lead to errors due to mismatches between stars.

Alternatively, it is possible to measure extinction without referring to a standard star by using a synthetic spectral energy distribution (SED) library and a numerical code that matches the available (spectro-)photometry to a grid with different stellar parameters (e.g. $T_{\text {eff }}, \log g$, and $Z$ ) and extinction amounts and laws (Maíz Apellániz 2004; Fitzpatrick \& Massa 2005). In this way, it is possible to derive the intrinsic or unextinguished SED directly from the data and, hence, calculate the extinction law directly by comparison with the observed spectral fluxes. Here we will follow that approach for the optical/NIR extinction using CHORIZOS (Maíz Apellániz 2004, 2005a), a Bayesian code that allows the fitting of (spectro-)photometric data to an SED grid of up to five dimensions, including parameterized extinction laws $^{2}$. For the amount of extinction CHORIZOS uses as parameter $E(4405-5495) \equiv A(4405)-A(5495)$, the monochromatic equivalent to $E(B-V)$, because the former depends only on the

${ }^{2}$ CHORIZOS is a public sofware written in IDL that can be downloaded from http://jmaiz.iaa.es 


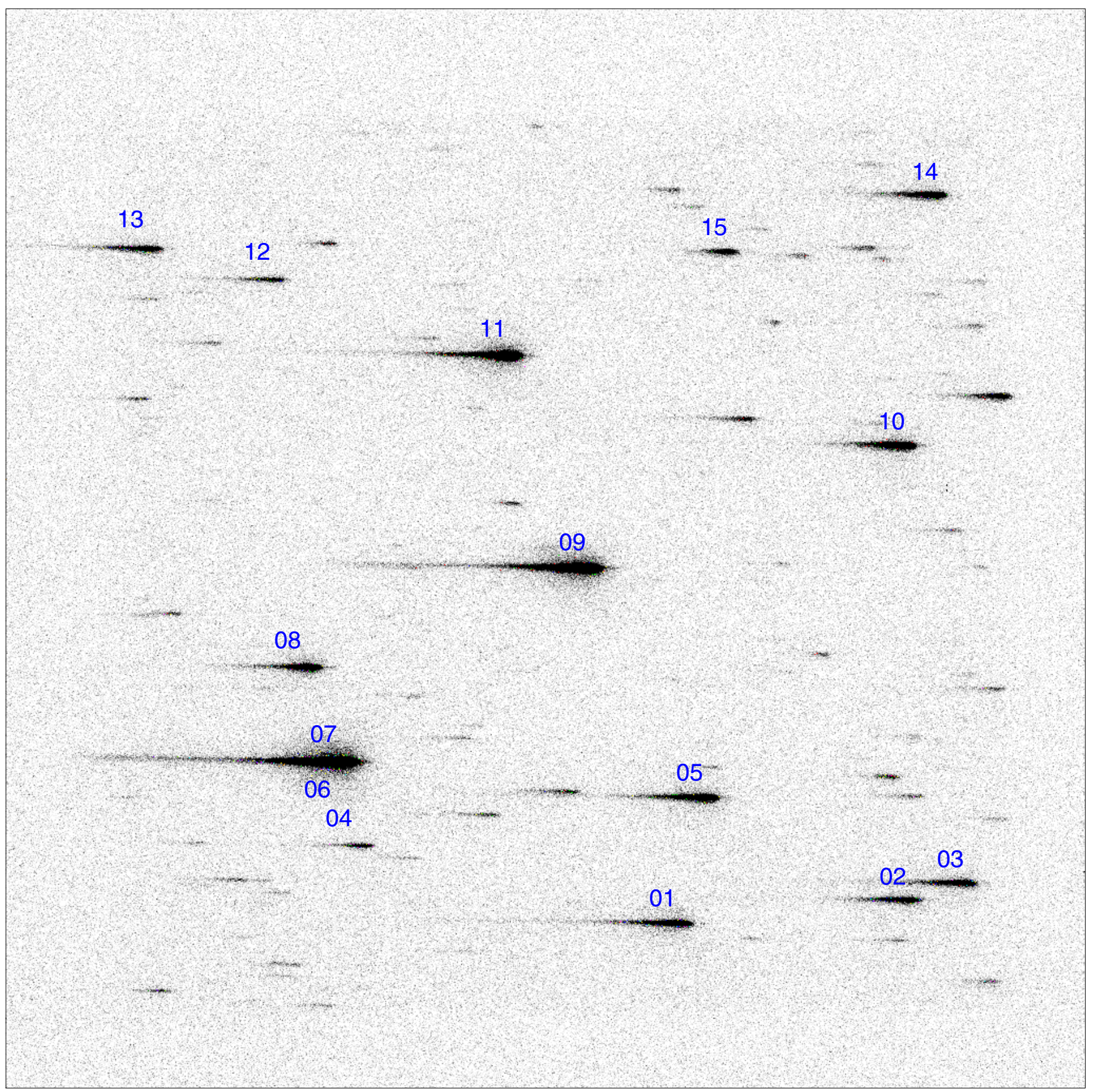

Fig. 2. STIS NUV-MAMA objective prism exposure of the SMC B1-1 region. See Fig. 1 for orientation and field size.

amount and type of dust while the latter also depends on the spectral type of the source ${ }^{3}$. In other words, the same amount and type of dust in front of an $\mathrm{O}$ star and an $\mathrm{M}$ star will produce the same $E(4405-5495)$ but different $E(B-V)$, so using $E(B-V)$ as an indicator of the amount of extinction can lead to some counterintuitive results (see e.g. Massey et al. 2005) and should be avoided to characterize an extinction law.

In order to use CHORIZOS to derive the intrinsic SED of the UV-bright objects in SMC B1-1 it is useful to take into account different considerations. First of all, optical-NIR extinction laws have relatively simple functional forms and

\footnotetext{
3 Throughout this work, wavelengths will be expressed in $\AA$ unless otherwise explicitly stated.
}

the Galactic laws can in principle be accurately described using a single-family parameter (Cardelli et al. 1989). In this wavelength range the published SMC extinction laws are not too different from the Galactic ones ${ }^{5}$ : for example, the SMCbar law of Gordon \& Clayton (1998) is quite similar to the Cardelli et al. (1989) law with $R_{5495}=2.8$ for $\lambda>3000 \AA$ $\left(R_{5495} \equiv A(5495) / E(4405-5495)\right.$ is the monochromatic

${ }^{4}$ Recent works have determined that there may be more variations than previously expected in the NIR extinction law but the effect of those variations should be relatively small in our case since, as we will later see, our targets have all $A_{K}<0.2 \mathrm{mag}$.

5 The outstanding exception being that of $\mathrm{AzV}$ 23/Sk 17, but see below. 
equivalent to $R_{V}$ ). See also Maíz Apellániz et al. (2012, in prep.). In the UV, the extinction morphology is more complicated and, as Fitzpatrick \& Massa (2007) have shown, cannot be accurately described by a single-parameter family. Therefore, it makes sense to derive the intrinsic SEDs by excluding the photometric data with the shortest wavelengths because of the likely confusion between intrinsic (e.g. $T_{\text {eff }}$ ) and extinction-law effects. Consequently, we will use the longest-wavelength UV photometric data (F25CN270) combined with the optical (UBVI) and NIR $(J H K)$ data for that purpose. Second, given the location in the SMC and the observed magnitudes, we expect our stars to be early-to-mid B main-sequence stars with metallicity near $\log Z=-1.0$. Also, given that the optical-NIR colors of nonsupergiant B stars are relatively insensitive to gravity and metallicity, it will be appropriate to constrain our synthetic SED grid to main-sequence models with $\log Z=-1.0$ and to use $T_{\text {eff }}$, $E(4405-5495)$, and extinction law type as the free parameters in the grid. Note that the best photometric discriminant for the effective temperature of B stars in the optical-NIR range is the Balmer jump and that with our filter selection we have two photometric points to its left (F25CN270 and $U$ ) and two points to its right $(B$ and $V)$, which allows us to measure the temperature precisely. Finally, in order to use consistent zero-points for the different photometric systems involved, we will use the Johnson sensitivity curves as determined by Maíz Apellániz (2006) and the zero-point system of Maíz Apellániz (2007).

Taking into account the considerations in the previous paragraph, we executed an initial CHORIZOS run for each of the 14 point-like visible sources using as input the F25CN270+ $U B V I+J H K$ photometry and as SEDs Kurucz atmospheres with main-sequence gravities and $\log Z=-1.0$ and Cardelli et al. (1989) extinction laws, leaving three free parameters to fit ( $T_{\text {eff }}, E(4405-5495)$, and $\left.R_{5495}\right)$. The results of that initial run show that all 14 sources have $E(4405-5495)$ lower than 0.60 and only three have values above 0.25 . Given the low extinction of the 11 sources with $E(4405-5495)<0.25$, their values of $R_{5495}$ are mostly unconstrained, as expected if the spectral types are not known a priori. For the three more extinguished stars we derive values of $R_{5495}$ between 2.9 and 3.5 with uncertainties of $\approx 0.3$ i.e. similar to those of the average Galactic and SMC extinction laws. This result prompted us to do a second and final CHORIZOS run with the same photometric data and parameters but using a fixed extinction law, the SMC-bar of Gordon \& Clayton (1998). The obtained values for $T_{\text {eff }}$ and $E(4405-5495)$ in the final CHORIZOS run are similar to those of the first one but with slightly lower error bars. The results of the second run are shown in Table 2 and plotted (for the four stars with the highest mean values of $E(4405-5495))$ in Figs. 3 and 4. As expected, all stars have temperatures consistent with being B stars ${ }^{6}$. Their extinction-corrected luminosities are all consistent with being main-sequence stars.

\subsection{Spectral extraction}

To extract the multiple spectra present in the objective prism data (Fig. 2) we used MULTISPEC (Maíz Apellániz 2005b), a software package specifically designed for the extraction of multiple spectra from slitless HST exposures of crowded fields ${ }^{7}$.

\footnotetext{
${ }_{6}$ Very recently, Maíz Apellániz et al. (2012, in prep.) have derived a new optical/NIR extinction law for 30 Doradus. Using that extinction law does not introduce fundamental changes to the results shown here.

7 MULTISPEC is a public sofware written in IDL that can be downloaded from http://jmaiz.iaa.es
}

Table 2. Second-run CHORIZOS results.

\begin{tabular}{lccc}
\hline \hline Star & $T_{\text {eff }}$ & $E(4405-5495)$ & Reduced $\chi_{\text {min }}^{2}$ \\
\hline 01 & $13600 \pm 1300 \mathrm{~K}$ & $0.14 \pm 0.05$ & 1.1 \\
02 & $12600 \pm 900 \mathrm{~K}$ & $0.03 \pm 0.03$ & 6.8 \\
03 & $12600 \pm 1100 \mathrm{~K}$ & $0.06 \pm 0.04$ & 1.8 \\
04 & $13500 \pm 3800 \mathrm{~K}$ & $0.19 \pm 0.15$ & 0.2 \\
05 & $11900 \pm 800 \mathrm{~K}$ & $0.17 \pm 0.03$ & 3.7 \\
$06+07$ & $13700 \pm 700 \mathrm{~K}$ & $0.10 \pm 0.03$ & 3.8 \\
08 & $23500 \pm 2900 \mathrm{~K}$ & $0.51 \pm 0.04$ & 1.5 \\
09 & $16900 \pm 1300 \mathrm{~K}$ & $0.20 \pm 0.03$ & 2.1 \\
10 & $15600 \pm 1300 \mathrm{~K}$ & $0.38 \pm 0.03$ & 4.8 \\
11 & $19000 \pm 1900 \mathrm{~K}$ & $0.27 \pm 0.04$ & 2.8 \\
12 & $12400 \pm 3100 \mathrm{~K}$ & $0.14 \pm 0.04$ & 3.2 \\
13 & $10400 \pm 800 \mathrm{~K}$ & $0.09 \pm 0.06$ & 2.2 \\
\hline
\end{tabular}

Notes. Results for stars 14 and 15 are not shown beacuse of the poor quality of the fits.

MULTISPEC works by fitting multiple spatial profiles for each column in the spectral exposure. We used v2.0 of the code, which allows the use of tabulated spatial profiles and which has been applied by Knigge et al. (2008) to extract UV spectra from STIS/FUV-MAMA G140L exposures of 47 Tuc. For the flux calibration of the data we used Maíz Apellániz \& Bohlin (2005) and included time-dependent sensitivity corrections.

The extracted spectra for stars 08, 09, 10, and 11 are shown in Fig. 4 along with the mode CHORIZOS SED, which assumes a Gordon \& Clayton (1998) SMC-bar extinction law. The extracted spectra have been binned in wavelength to obtain a uniform signal-to-noise ratio $(\mathrm{S} / \mathrm{N})$ in each bin. The observed and model spectra have relatively similar values of $f_{v}$ in their common wavelength range, indicating that the extinction law for those stars cannot be too different from the SMCbar one of Gordon \& Clayton (1998). Note, however, that there are some readily apparent differences: stars 08 and 11 show a weak $2175 \AA$ absorption structure and the measured UV fluxes for stars 09 and 10 are higher than the model ones.

\subsection{Source selection}

As previously mentioned, only three point sources $(08,10$, and 12) have $E(4405-5495)>0.25$ and a fourth one (09) has a slightly lower value. Those will be the four sources for which we will calculate the UV extinction law because for the rest the relative uncertainties on $k(\lambda)$ are too large to yield a useful result. Note that previous studies have derived extinction laws for stars with even lower values of $E(4405$ - 5495), but that was possible because of the availability of data with higher $\mathrm{S} / \mathrm{N}$ than we are using here.

\subsection{UV extinction-law calculation}

Finally, we obtain the UV extinction law $k(\lambda)$ which, following the usual practice, we normalize as:

$$
k(\lambda)=k(5495)+\frac{E(\lambda-5495)}{E(4405-5495)}
$$

and then consider only the second term, which is 0 for $\lambda=5495 \AA$ and 1 for $\lambda=4405 \AA$. We recall that we used monochromatic rather than filter-integrated values for the definition of the extinction law. The mode value of $k(\lambda)-k(5495)$ is obtained by dividing the extracted spectrum for each star by the unextinguished version of the CHORIZOS-derived mode SED and 

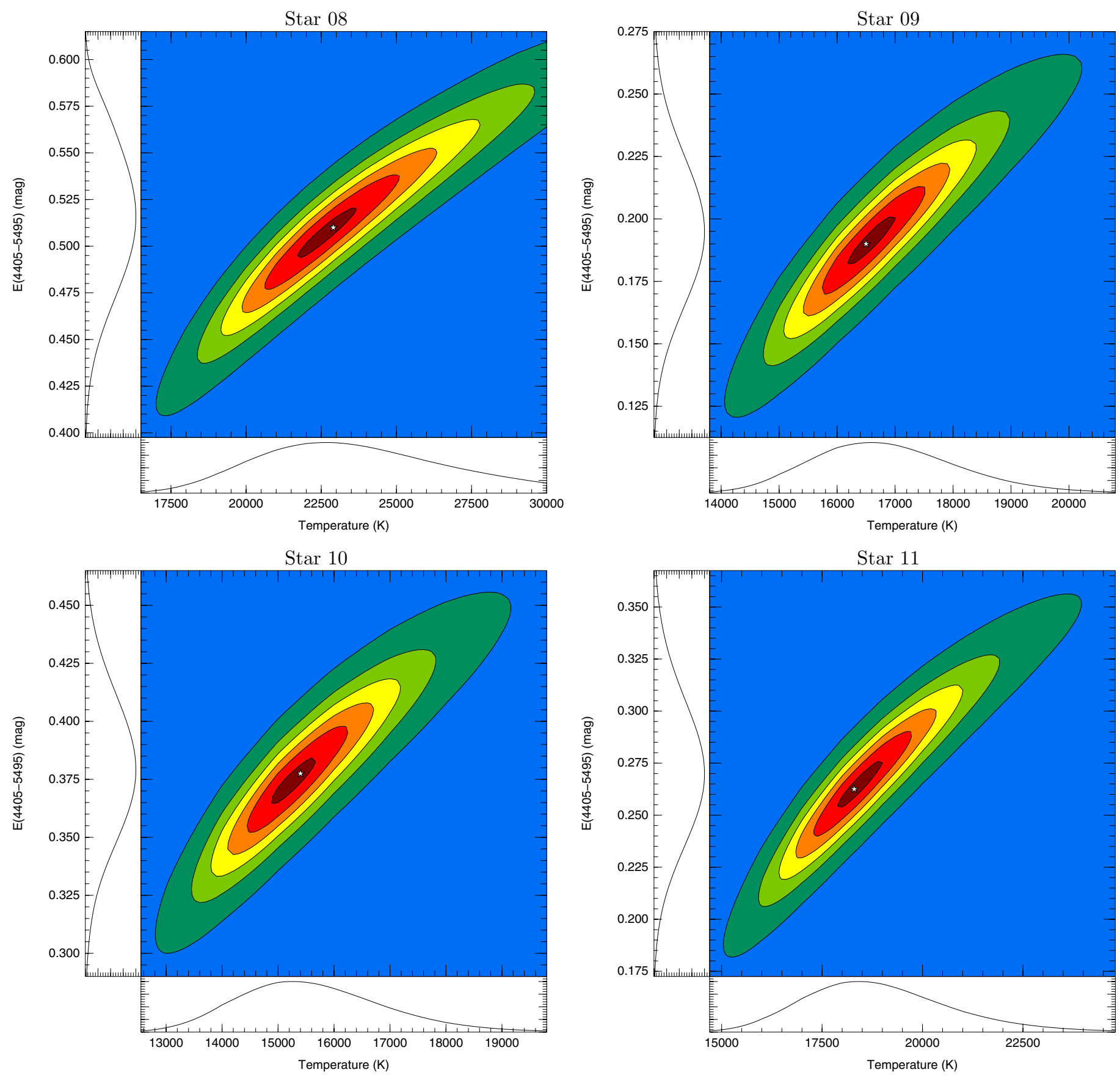

Fig. 3. Contour plots showing the likelihood in the $T_{\text {eff }}-E(4405-5495)$ plane for the four stars in the second CHORIZOS run with the highest mean value for $E(4405-5495)$. The contour intervals are linearly spaced with the minimum and maximum at 0.05 and 0.95 times the peak (mode) value, respectively. The star shows the location of the mode (not the mean) and the plots on each axis show the integrated likelihood for that parameter.

normalizing the result. A similar procedure can be performed for the filter-integrated quantities derived from the measured F25QTZ and F25CN182. Both spectral and photometric results are shown in Fig. 5. One readily apparent result is that there is a decrease in the intensity of the $2175 \AA$ absorption structure as we follow the stellar sequence $11 \rightarrow 08 \rightarrow 09 \rightarrow 10$.

One practical way of characterizing the UV extinction law is by using the six-parameter $\left(c_{1}, c_{2}, c_{3}, c_{4}, x_{0}, \gamma\right)$ Fitzpatrick \& Massa (1990) recipe, which gives

$k(\lambda)-k(5495)=c_{1}+c_{2} x+c_{3} D\left(x, x_{0}, \gamma\right)+c_{4} F(x)$, where $x=1 / \lambda$ (usually expressed in $\mu^{-1}$ ),

$$
D\left(x, x_{0}, \gamma\right)=\frac{x^{2}}{\left(x^{2}-x_{0}^{2}\right)^{2}+x^{2} \gamma^{2}},
$$

and

$$
F(x)= \begin{cases}0.5392(x-5.9)^{2}+0.05644(x-5.9)^{3}, & x>5.9 \\ 0, & x \leq 5.9 .\end{cases}
$$

We wrote a $\chi^{2}$-minimization code to fit such a Fitzpatrick \& Massa (1990) six-parameter function to the spectral (1700$2900 \AA$ A ) and photometric (F25QTZ and F25CN182) data for 
J. Maíz Apellániz and M. Rubio: UV extinction toward a quiescent molecular cloud in the SMC
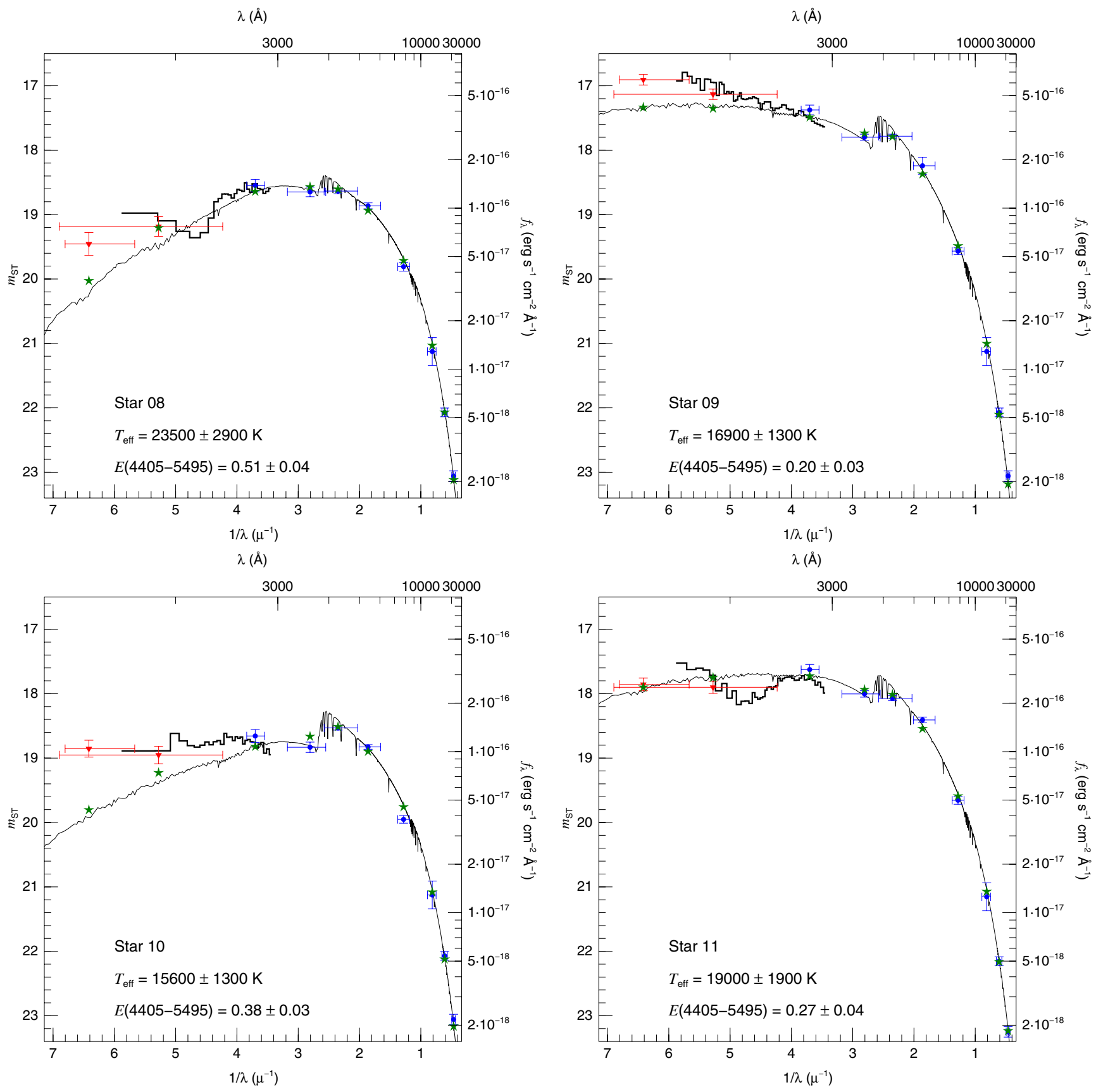

Fig. 4. MULTISPEC and fixed extinction-law CHORIZOS results for the four stars with the highest $E(4405-5495)$. The red and blue data points with error bars correspond to the observed UV-optical-NIR photometric magnitudes, with the vertical error bars showing the uncertainties and the horizontal ones the approximate extent of the filters. Only the blue data points points were used for the CHORIZOS fits. The continuous FUV-toNIR line shows the CHORIZOS mode SED, which assumes a Gordon \& Clayton (1998) SMC-bar extinction law throughout the full wavelength range, while the NUV-only histogram shows the MULTISPEC extraction. The green stars show the synthetic photometry that corresponds to the CHORIZOS mode SED. The CHORIZOS values for $T_{\text {eff }}$ and $E(4405-5495)$ are given for each star.

each of the four stars with higher extinction. When doing so, one has to be careful to account not only for random errors arising from the finite $\mathrm{S} / \mathrm{N}$ of the data but also for systematic ones arising from possible spectral mismatches (in our case, this corresponds to the uncertainty in the $T_{\text {eff }}$ and $E(4405-5495)$ values derived by CHORIZOS). The results are shown and plotted in Fig. 5.

As previously noted, there is a sequence of intensities of the $2175 \AA$ absorption structure (measured by $c_{3}$ ) in the order $11 \rightarrow 08 \rightarrow 09 \rightarrow 10$. It should be also pointed out that the values of the parameter that measure the FUV slope $\left(c_{4}\right)$ are anomalously high. However, those values are not very significant (their errors are very large) because they are based mostly on a single photometric point (F25QTZ) centered at relatively long FUV wavelengths.

For our calculations we did not perform a prior subtraction of the Galactic contribution to the extinction. As described by Zagury (2007), for the Magellanic Clouds this cannot be done from the data itself unless one assumes a smooth spatial distribution of the Galactic cirri, something that the MIR observations do not support. Therefore, the UV extinction laws derived here are in principle a combination of a Galactic and an 
$\lambda(\AA)$

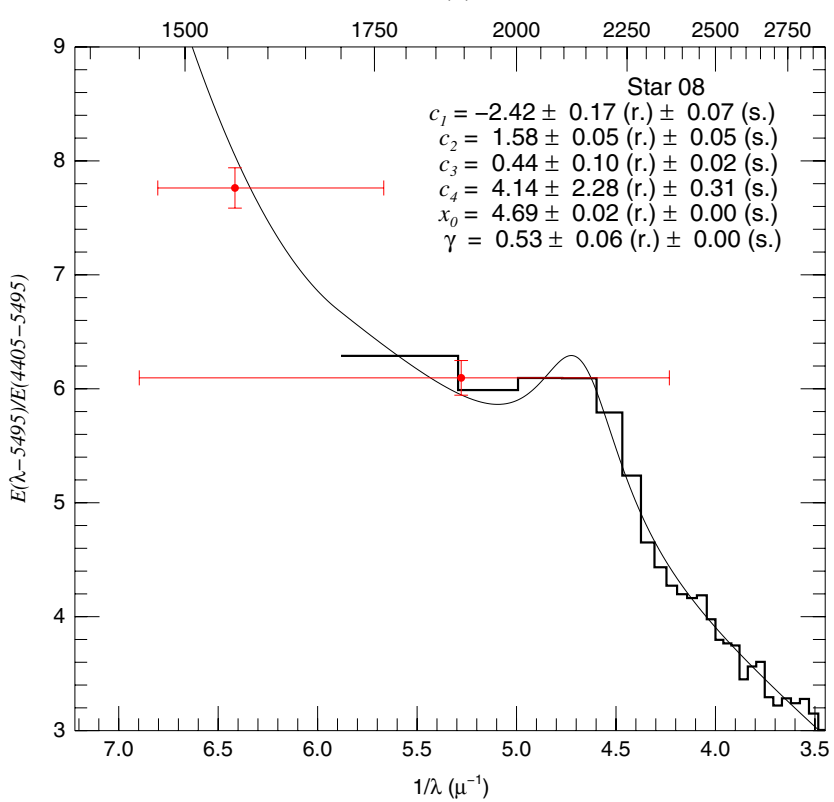

$\lambda(\AA ̊)$

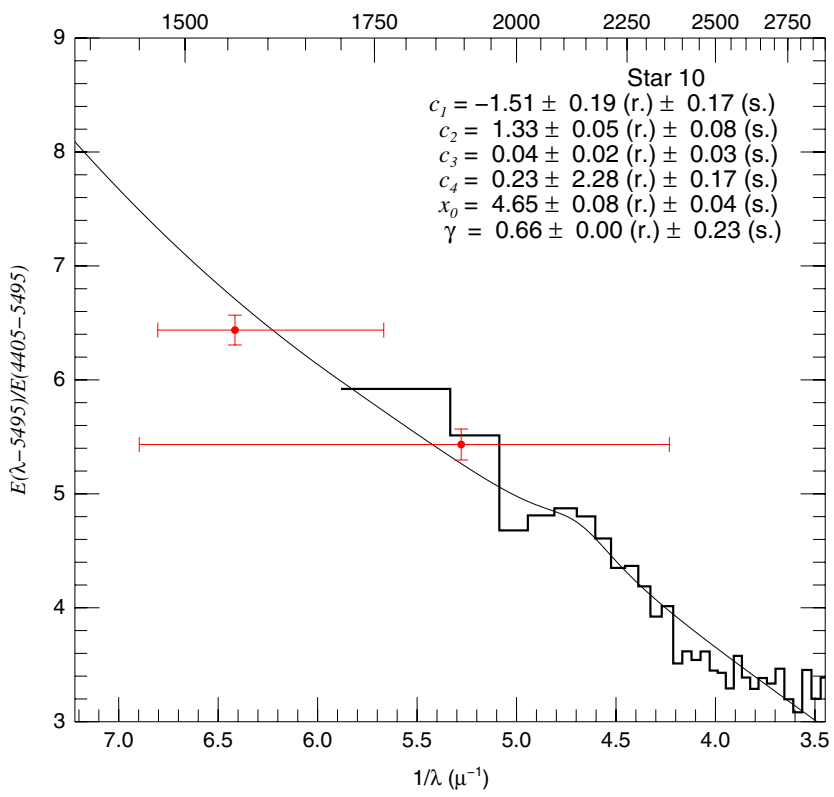

$\lambda(\AA)$

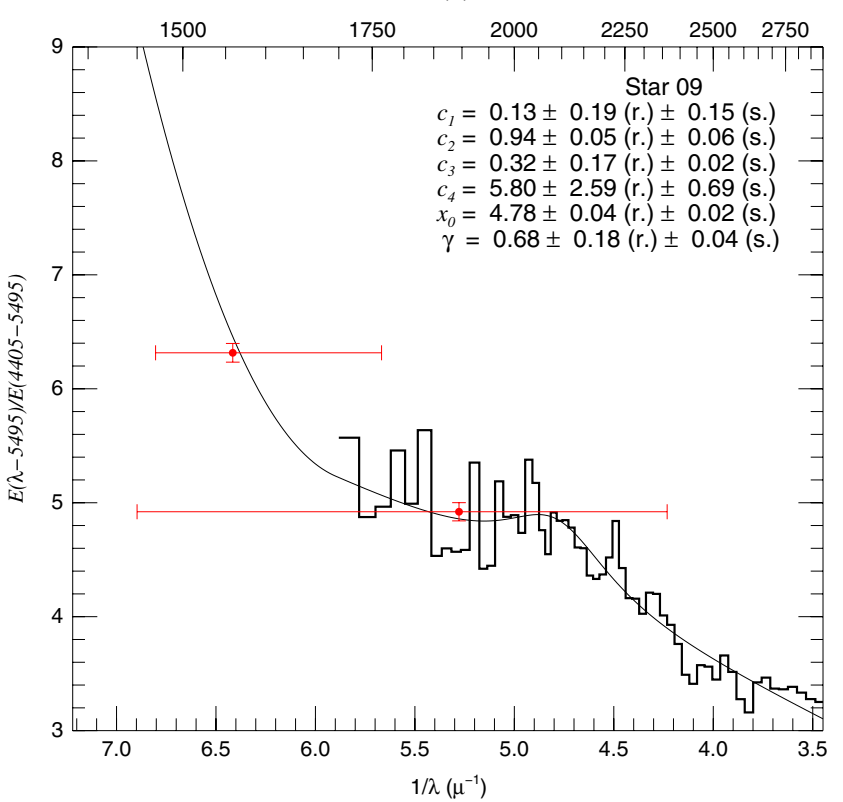

$\lambda(\AA)$

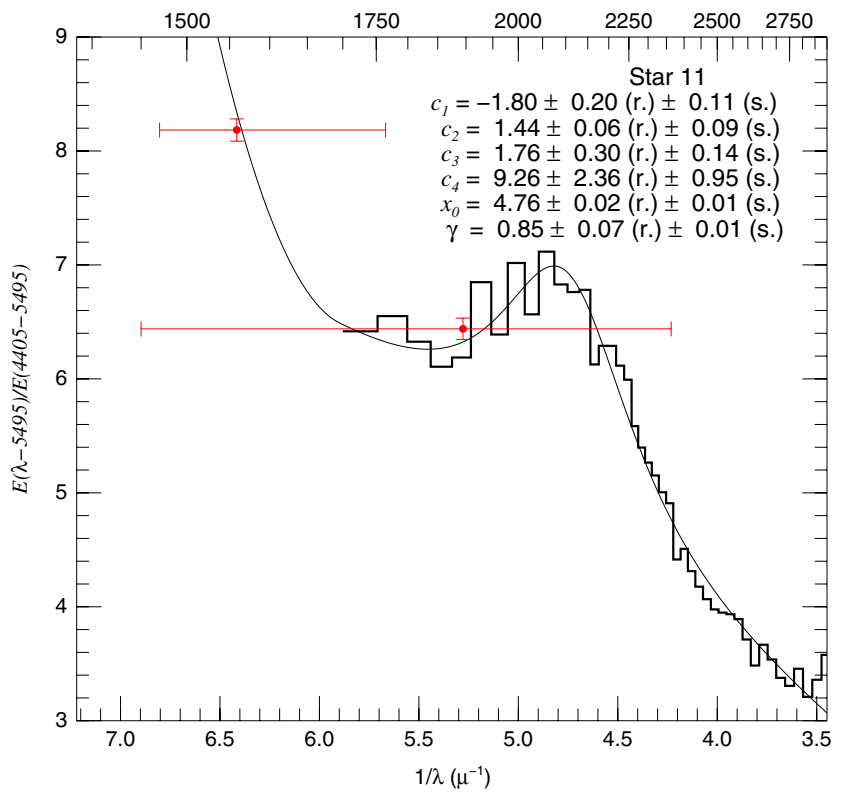

Fig. 5. Observed extinction laws (histogram used for the spectral data, points with error bars for the photometric data) and fitted Fitzpatrick \& Massa (1990) extinction laws (continuous line) for each of the four stars in Fig. 4. The values of the fitted parameters are given in each case with their random and systematic errors indicated by "r." and "s.", respectively.

SMC components. However, the existence of stars with very low extinction in the vicinity [e.g. star 02 has $E(4405-5495)=$ $0.03 \pm 0.03$ ] argues in favor of the dominance of the SMC component. An additional point against the importance of a Galactic dust component is the wide range in reddenings in our sample, with values from 0.03 to $0.51 \mathrm{mag}$. Note that at a distance of e.g. $1 \mathrm{kpc}$ to a hypothetical Galactic cloud, $10^{\prime \prime}$ correspond to just 10000 AU. To our knowledge, no Galactic cirrus shows such large variations on such small scales. The low value of $E(4405-5495)$ for star 02 also agrees with the foreground reddening of 0.037 measured by Schlegel et al. (1998) and with the more recent work of Subramaniam \& Subramanian (2010), who found that most of the SMC has a Galactic foreground extinction lower than $A_{V}=0.1 \mathrm{mag}$.

\section{Looking back at the five SMC stars with preexisting extinction laws}

To check that our methods are consistent with previous works, we used CHORIZOS to calculate the values of $E(4405-5495)$ and $R_{5495}$ for the five SMC stars with preexisting extinction laws. For that purpose, we used the Gordon et al. (2003) $U B V R I$ and the 2MASS $J H K_{\mathrm{S}}$ photometry for those stars $^{8}$. In this case we used a newer version of CHORIZOS that allows the stellar parameters to be $T_{\text {eff }}$, luminosity class, and metallicity. By luminosity class, we mean a quantity that runs from 0.0 (hypergiants)

8 They are much brighter than the SMC B1-1 stars because they are supergiants. Hence, they all have 2MASS photometry with small uncertainties. 
J. Maíz Apellániz and M. Rubio: UV extinction toward a quiescent molecular cloud in the SMC

Table 3. CHORIZOS results for the five stars with preexisting UV extinction laws.

\begin{tabular}{lcccccc}
\hline \hline Star & $T_{\text {eff }}$ & Luminosity class & $R_{5495}$ & $E(4405-5495)$ & $A_{V}$ & Red. $\chi_{\text {min }}^{2}$ \\
\hline AzV 18/Sk 13 & $19000 \mathrm{~K}$ & $1.19 \pm 0.01$ & $3.93 \pm 0.64$ & $0.178 \pm 0.022$ & $0.696 \pm 0.040$ & 1.9 \\
AzV 23/Sk 17 & $18000 \mathrm{~K}$ & $1.01 \pm 0.01$ & $3.41 \pm 0.08$ & $0.253 \pm 0.003$ & $0.878 \pm 0.015$ & 1.9 \\
AzV 214 & $23000 \mathrm{~K}$ & $1.80 \pm 0.03$ & $3.09 \pm 0.20$ & $0.246 \pm 0.007$ & $0.772 \pm 0.034$ & 2.2 \\
AzV 398 & $28000 \mathrm{~K}$ & $1.93 \pm 0.03$ & $3.57 \pm 0.28$ & $0.322 \pm 0.018$ & $1.162 \pm 0.036$ & 1.3 \\
AzV 456/Sk 143 & $28000 \mathrm{~K}$ & $1.19 \pm 0.02$ & $2.63 \pm 0.14$ & $0.337 \pm 0.011$ & $0.901 \pm 0.027$ & 0.6 \\
\hline
\end{tabular}

to 5.5 (ZAMS) and, for a given $T_{\text {eff }}$ and metallicity, yields a luminosity similar to the one derived from spectral classification (with $\mathrm{Ia}+$ translated as 0.0 and $\mathrm{Vz}$ as 5.5). The SEDs in this case were TLUSTY (Lanz \& Hubeny 2003, 2007). Three parameters were left fixed: $T_{\text {eff }}$ (from the spectral type), metallicity (SMC), and the logarithm of the distance (4.782). Three parameters were allowed to vary: Luminosity class, $R_{5495}$, and $E(4405-5495)$. For $\mathrm{AzV} 398$ we excluded the $K_{\mathrm{s}}$ photometry from the fit after an initial run because the star appears to have an excess in that band compared to the TLUSTY models, likely a wind effect. The Cardelli et al. (1989) extinction laws were used. Results are shown in Table 3. Note that the values there for $R_{5495}$ and $E(4405-5495)$ are monochromatic quantities while $A_{V}$ is filter-integrated.

The reduced $\chi_{\min }^{2}$ in all cases is quite good, indicating the good quality of the photometry. The (photometry-derived) luminosity classes are all between 1.0 and 2.0, as expected for supergiants. Our results for $E(4405-5495)$ and $R_{5495}$ differ significantly from the band-integrated ones $[E(B-V)$ and $R_{V}$, respectively] of Gordon et al. (2003). The differences in $E(4405-5495)$ likely arise because we are measuring absolute extinctions while they were measuring extinctions relative to a comparison star. Indeed, our values for $E(4405-5495)$ are higher than their values of $E(B-V)$ by 0.01 to 0.10 mag, as expected under these circumstances. However, we also measure values of $R_{5495}$ that are systematically higher by $\sim 0.6$. That is harder to assign to the inclusion of an additional $R_{5495}=3.1$ component in our values (the one responsible for the additional extinction), since that would pull them toward 3.1 and not increase all of them independently of $R_{5495}$. The difference may be attributed to the different methodologies and to the fact that our quantities are monochromatic and theirs are band-integrated.

The uncertainties in Table 3 (and in Table 2 as well) are formal (or random) ones. There are also systematic uncertainties caused by the mismatch between the SEDs used and the real ones and due to the problems with the Cardelli et al. (1989) extinction laws (fundamentally, the use of a seventhdegree polynomial as a functional form in the optical range, see Maíz Apellániz et al. 2007; Maíz Apellániz \& Sota 2008; Maíz Apellániz et al. 2012, in prep.). However, an analysis of the photometric residuals indicates that the Balmer jump seems to be well fit. Furthermore, these objects have $E(4405-5495)<0.5$, so the systematic effects of the Cardelli et al. (1989) extinction laws should be small ${ }^{9}$. Therefore, we do not expect the systematic uncertainties to be larger than the random ones, especially for $R_{5495}$.

The weighted mean of the $R_{5495}$ values for the four SMC bar stars is $3.36 \pm 0.07$ and the results are consistent with the four real $R_{5495}$ values being the same (three cases within one sigma, one between one and two sigma). Therefore, the results for those

\footnotetext{
9 A quite different situation compared to the more extinguished case of Pismis 24-1 case discussed in Maíz Apellániz et al. (2007).
}

four stars and the three more extinguished stars in SMC B1-1 are consistent with a visible-NIR extinction law not too different from the typical Galactic one, which has $R_{5495}=3.1-3.2$. On the other hand, the result for AzV 456/Sk 143 does indeed seem different, since its $R_{5495}$ is $\sim 5$ sigmas lower.

\section{Discussion}

In this paper we have calculated for the first time the UV extinction law for four stars in SMC B1-1, a quiescent cloud in the Small Magellanic Cloud. For those stars and for the other five stars with previously existing UV extinction laws we have also calculated their $E(4405-5495)$ and $R_{5495}$ from visibleNIR photometry.

For the four stars in SMC B1-1. all located within a few pc of each other, we found significant variations in the extinction law and values. The latter is simply a manifestation of the small-scale structure in the dust distribution while the former implies a different dust composition. The most clear variations in the UV extinction law are in the strength of the $2175 \AA$ bump, which goes from non-existing (as is the case for the four of the SMC bar stars with preexisting laws) to a case of moderate strength by Galactic standards though still weaker than the previously studied SMC bar star $\left(c_{3}=1.76 \pm 0.30\right.$ for the new target, $2.57 \pm 0.22$ for the old one). $c_{3}$ does not appear positively correlated with $E(4405-5495)$ (the most and the least extinguished stars have weak bumps while the two stars with intermediate extinctions represent the extremes in bump strength), which argues against a foreground Galactic cloud as the source of the extinction.

Despite the significant UV variations, the SMC visibleNIR extinction law appears to be more uniform, at least within the current measurement errors. The variations in $R_{5495}$ have been attributed to different dust grain sizes for a long time (Baade \& Minkowski 1937). All observed eight SMC bar stars with well-measured $R_{5495}$ have values compatible with the typical Galactic one and only the SMC star measured in the wing has a somewhat lower $R_{5495}$. However, there are no clear cases of high values $\left(R_{5495} \sim 5\right)$, such as those found in some Galactic H II regions (see e.g. Arias et al. 2006). That is not surprising, given that the Galactic cases with large $R_{5495}$ are found within the bright areas of their $\mathrm{H}_{\mathrm{II}}$ regions and that is not the case for any of the analyzed SMC stars ${ }^{10}$.

The existence of the $2175 \AA$ bump in stars located in relatively quiescent regions of the SMC argues in favor of its absence being caused by the presence of intense UV radiation fields and stellar winds, which would destroy its carrier (see Clayton et al. 2003; Sofia et al. 2006). Nevertheless, our results also suggest that the final explanation may be more complex. Otherwise, the four stars in SMC B1-1 would show the bump in their extinction laws. Also, since extinction appears to be patchy and

${ }_{10}$ AzV 214 is in NGC 346 but outside the central bright nebulosity. 
associated with local dust clouds, $c_{3}$ should be correlated with $E(4405-5495)$. Neither of those two effects appear in on our results.

Acknowledgements. This article is based on observations made with the NASA/ESA Hubble Space Telescope (HST) associated with GO program 9718 and obtained at the Space Telescope Science Institute, which is operated by the Association of Universities for Research in Astronomy, Inc., under NASA contract NAS 5-26555. Support for both authors was provided by the Chilean FONDECYT grant number 1080335. J.M.A. also acknowledges support from [a] the Spanish Government Ministerio de Educación y Ciencia through grants AYA2004-08260-C03, AYA2004-05395, AYA2007-64712, AYA200764052, and AYA2010-17631 and the Ramón y Cajal Fellowship program, cofinanced with FEDER funds, and [b] the Consejería de Educación of the Junta de Andalucía through grants TIC-101 and P08-TIC-4075. M.R. is also supported by the Chilean Center for Astrophysics FONDAP grant number 15010003. The authors would like to thank the referee, Geoff Clayton, for his suggestions in improving the paper, and François Boulanger for his inspiration and support in the early stages of this project.

\section{References}

Arias, J. I., Barbá, R. H., Maíz Apellániz, J., Morrell, N. I., \& Rubio, M. 2006, MNRAS, 366, 739

Baade, W., \& Minkowski, R. 1937, ApJ, 86, 123

Cardelli, J. A., Clayton, G. C., \& Mathis, J. S. 1989, ApJ, 345, 245

Clayton, G. C., Wolff, M. J., Sofia, U. J., Gordon, K. D., \& Misselt, K. A. 2003, ApJ, 588, 871

Fitzpatrick, E. L., \& Massa, D. 1990, ApJS, 72, 163

Fitzpatrick, E. L., \& Massa, D. 2005, AJ, 130, 1127

Fitzpatrick, E. L., \& Massa, D. 2007, ApJ, 663, 320

Gordon, K. D., \& Clayton, G. C. 1998, ApJ, 500, 816
Gordon, K. D., Clayton, G. C., Misselt, K. A., Landolt, A. U., \& Wolff, M. J. 2003, ApJ, 594, 279

Knigge, C., Dieball, A., Maíz Apellániz, J., et al. 2008, ApJ, 683, 1006

Lanz, T., \& Hubeny, I. 2003, ApJS, 146, 417

Lanz, T., \& Hubeny, I. 2007, ApJS, 169, 83

Maíz Apellániz, J. 2004, PASP, 116, 859

Maíz Apellániz, J. 2005a, in The Three-Dimensional Universe with Gaia, ed. C. Turon, K. S. O'Flaherty, \& M. A. C. Perryman, ESA SP, 576, 449

Maíz Apellániz, J. 2005b, STIS Instrument Science Report 2005-02 (STScI: Baltimore)

Maíz Apellániz, J. 2006, AJ, 131, 1184

Maíz Apellániz, J. 2007, in The Future of Photometric, Spectrophotometric and Polarimetric Standardization, ed. C. Sterken, ASP Conf. Ser., 364, 227

Maíz Apellániz, J., \& Bohlin, R. C. 2005, STIS Instrument Science Report 200501 (STScI: Baltimore)

Maíz Apellániz, J., \& Sota, A. 2008, in Rev. Mex. Astron. Astrofís. Conf. Ser., 33, 44

Maíz Apellániz, J., Walborn, N. R., Morrell, N. I., Niemelä, V. S., \& Nelan, E. P. 2007, ApJ, 660, 1480

Massa, D., Savage, B. D., \& Fitzpatrick, E. L. 1983, ApJ, 266, 662

Massey, P., Plez, B., Levesque, E. M., et al. 2005, ApJ, 634, 1286

Ochsenbein, F., Bauer, P., \& Marcout, J. 2000, A\&AS, 143, 23

Prévot, M. L., Lequeux, J., Maurice, E., Prévot, L., \& Rocca-Volmerange, B. 1984, A\&A, 132, 389

Rubio, M., Boulanger, F., Rantakyro, F., \& Contursi, A. 2004, A\&A, 425, L1

Schlegel, D. J., Finkbeiner, D. P., \& Davis, M. 1998, ApJ, 500, 525

Sofia, U. J., Gordon, K. D., Clayton, G. C., et al. 2006, ApJ, 636, 753

STScI 2007, STIS Instrument Handbook, ed. J. Kim Quijano, S. Hoifeltz, \& J. Maíz Apellániz

Subramaniam, A., \& Subramanian, S. 2010, Interstellar Matter and Star Formation: A Multi-wavelength Perspective, ed. D. K. Ojha, ASI Conf. Ser., 1,107

Zagury, F. 2007, Ap\&SS, 312, 113

Zaritsky, D., Harris, J., Thompson, I. B., Grebel, E. K., \& Massey, P. 2002, AJ, 123,855 